\title{
Seven day service policy lacked transparency, review concludes
}

In this News article (BMJ 2016;355:i6308, doi:10.1136/bmj. i6308), the first sentence of the fourth paragraph should read, "The report examined 79 government policies from 13 departments published between May 2015 and May 2016, including six policies from the Department of Health for England." 